\title{
Evaluation of Morphology of Premature Ventricular Contraction on 12-Lead Electrocardiogram
}

\author{
Umme Habiba Ferdaushi ${ }^{1}$, M. Atahar Ali ${ }^{2}$, Shaila Nabi ${ }^{3}$, Mainul Islam4, Md. Shamshul Alam ${ }^{5}$, Md. Arifur Rahman 6
}

\begin{abstract}
:
Background-Evaluation of different morphology of premature ventricular contraction (PVC) in 12-lead ECG might reflect the presence or absence of myocardial diseases and determine PVC foci. It is important for ablation procedure and it can help in pre-procedural planning and potentially may improve ablation outcome.

Methods and Results-In this study, 12-lead Electrocardiogram (ECG) of 50 patients with or without structural cardiac diseases, who had experienced PVC, were obtained. PVC QRS duration, contour, pattern, unifocal or multifocaland different morphology in various lead were evaluated. PVC-QRS morphology of 50 ECGs showed QRSd d" $140 \mathrm{~ms}$ was $60 \%$, >140ms was $24 \%$, $>160 \mathrm{~ms}$ was $16 \%$. QRS notching $<40 \mathrm{~ms}$ was $42 \%$, $>40 \mathrm{~ms}$ was $16 \%$, smooth contour was $42 \%$. The morphology of PVCs in lead V1, RBBB morphology was $36 \%$, LBBB morphology was 64\%; in lead V1 \& V2, high r $8 \%$, low r $4 \%$. QRS wave polarity in lead I negative (QS, Qr, or rS wave pattern) $28 \%$, positive (R-wave pattern) $52 \%$; in lead II, III,
\end{abstract}

avF, positive $76 \%$. Of these RR' or Rr' pattern $20 \%$, R pattern $56 \%$. Negative $24 \%$. QRS transition in chest lead, $16 \%$ transition occur at V4-V5, 48\% at V3-V4, 4\% at V2-V3, 36\% at V1-V2 level. The pattern of PVCs were bigeminy $\mathbf{2 4} \%$, trigeminy $6 \%$, couplet $4 \%$, salvos $12 \%$, R on T $2 \%$, VT $6 \%$. Of the 32 PVCs originating from the RVOT, 8 were classified as of free-wall origin, 24 of septal, 14 of left, 26 of right, 4 of proximal, and 2 of distal origin. Of the 6 PVCs originating from the LVOT, 4 were originated from the LVOT close to the left coronary cusp and 2 were originated from the LVOT close to the right coronary cusp. Of the 12 PVCs originated from LV fascicle, 12 of posterior fascicle origin and none from anterior fascicle origin.

Conclusion-12-lead ECG is a simple, inexpensive and noninvasive tool to detect PVCs and facilitate their localization. By evaluating morphology of PVC, we can also predict the structural and functional state of heart.

Keywords: Electrocardiogram, Cardiac Arrhythmia, Premature Ventricular Beats,
Introduction:

Premature ventricular contraction (PVC) is the most common cardiac arrhythmia in patients with or without

1. Medical Officer, Department of Cardiology, National Institute of Cardiovascular Diseases, Dhaka, Bangladesh.

2. Professor of Cardiology, National Institute of Cardiovascular Diseases, Dhaka, Bangladesh.

3. Associate Professor, Department of Cardiology, National Institute of Cardiovascular Diseases, Dhaka, Bangladesh.

4. Assistnt Registrar, Department of Cardiology, National Institute of Cardiovascular Diseases, Dhaka, Bangladesh.

5. Associate Professor, Department of Cardiology, North Bengal Medical College \& Hospital, Sirajganj, Bangladesh.

6. Registrar, Department of Cardiology, National Institute of Cardiovascular Diseases, Dhaka, Bangladesh.

Address of Correspondence: Dr. Umme Habiba Ferdaushi, Medical Officer, Department of Cardiology, National Institute of Cardiovascular

Diseases, Dhaka, Bangladesh, E-mail: drhabibc30@yahoo.com any kind of diagnosed cardiac diseases ${ }^{1}$. PVC is an extra heart beat originates from the ventricles and comes before the normal heart beat. Although in general, this arrhythmia may occur in a healthy person, but it is more prevalent with increasing age and occur in association with a variety of cardiac and non-cardiac diseases such as hypertension, myocardial infarction, and so on ${ }^{2}$.

The mechanisms by which PVCs are generated include enhanced normal or abnormal automaticity, triggered activity resulting in afterdepolarizations and reentry. Several pattern of PVCs are described, like-bigeminy, trigeminy, quadrigeminy, couplet, salvos, unifocal, multifocal PVCs, $\mathrm{R}$ on $\mathrm{T}$ phenomenon, $\mathrm{VT}^{3}$. Today, the electrocardiogram (ECG) remains the simplest and cost 
effective non-invasive diagnostic method for determining arrhythmias. The morphological features of the premature ventricular complex (PVC) have been citedas a clue to the presence or absence of underlying cardiac disease ${ }^{4}$. Scherf and Schott ${ }^{5}$ recognized that PVCs with exceptionally wide QRS complexes frequently occurred in diseased hearts.Soloff ${ }^{4}$ found that PVCs with a bizarre and distorted configuration were highly suggestive of underlying myocardial disease in contrast to those with the "classic" smooth pattern. Morphology is also useful to localize the site of origin of PVCs before ablation procedure where indicated. This helps not only in preprocedural planning, but also can potentially improve ablation outcomes ${ }^{6}$.

PVCs may originate from various foci. If PVC focus is in right ventricle, it would appear as Left Bundle Branch Block (LBBB) and if it is in left ventricle, it would appear as Right Bundle Branch Block (RBBB) because in this state left ventricle would depolarize earlier. In general there are three common regions are defined for PVC foci: Right Ventricular Outflow Tract (RVOT), Left Ventricular Outflow Tract (LVOT) ${ }^{7}$ and Aortic Cusp (AC). Many researches for determining various divisions of idiopathic VT or PVC foci have been developed so far, including idiopathic ventricular tachycardia (IVT) consist of RVOT VT/PVC, Idiopathic Left Ventricular Tachycardia (ILVT), Idiopathic Propranolol sensitive VT (IPVT), LVOT VT/ $P V C[8]$ and $A C^{6}$. It has been reported that $60-80 \%$ of the idiopathic tachycardia in normal hearts arise from the RVOT and $10 \%$ of them arise from LVOT ${ }^{9}$. RVOTVT/PVC is more common in females at age 30 to 50 years old ${ }^{10}$ shows wide QRS complex and LBBB pattern with inferior axis ${ }^{11}$, whereas LVOT VT/PVC usually shows RBBB morphology in lead V1 with wide monophasic R-wave in precordial leads. Morphologic explanations of ECG characteristics are useful for differentiating of VT/PVC arising from the AC region. VT/PVC originated from the left coronary cusp produces multiphasic QRS morphology with an $\mathrm{M}$ or $\mathrm{W}$ configuration in lead $\mathrm{V} 1$ with a precordial transition no later than V2. A left bundle pattern with a wide small $R$ wave in lead V2 and a precordial transition usually at V3 is revealed in PVCs with a right coronary cusp origin ${ }^{6}$.

Kamakura et al. ${ }^{12}$ proposed the method to estimate the origin of VT/PVC from the RVOT and LVOT by using indexes obtained from 12-lead ECG. They classified PVC/ VT from the RVOT into 8 subdivisions by using 3dimensional anatomic relation: anteriorposterior, rightleft, and superior-inferior. The features they used for estimating the origin of PVC/VT consisted of morphology, amplitude, duration and polarity of QRS complex. To distinguish LVOT from RVOT region, they showed that R/ $S$ amplitude ratio in lead $V_{3}$ is a helpful index. If the ratio of R/S amplitude in $V_{3}$ is equal or higher than 1 , the PVC/ VT stems from LVOT zone, otherwise arises from RVOT.

The purpose of this study is to find out the various morphology and foci of PVCs using 12-lead ECG.

\section{Materials and methods:}

\section{Data collection}

12 lead ECGs of the 50 patients with or without structural cardiac disease, who had experienced PVC, were obtained. The data was collected from National Institute of Cardiovascular Diseases (NICVD) arrhythmia clinic.

\section{PVC detection}

First of all, PVCs were recognized and distinguished from the normal beats. Because of their greatness in height, depth and length, PVCs could easily be detected. They were characterized by-

- Duration of more than $120 \mathrm{msec}$

- Bizarre morphology that does not resemble usual aberration (i.e. a typical right or left bundle branch block).

- T wave in the opposite direction from the main QRS vector.

- A fully compensatory pause.

\section{QRS duration and morphology}

In this study we detected the duration, contour, pattern (bigemini, trigemini, quadrigeminy, couplet, triplet, salvoes), unifocal or multifocal and various morphology of PVCs in different leads like lead I,II,III,avF,V1 and QRS transition in chest lead.

Presence or absence of notching in PVC was also detected. Notching in QRS complex was determined as a tri-phasic $\mathrm{R}$ or $\mathrm{Q}$ wave with an interval greater than 40 msec between the first and second peak of the QRS complex. Existence of notching is considered when notching is observed in more than three of the six limb leads. When notching occurred near the summit, the non-dominant peak was measured.

\section{Classification of anatomical site of PVCs by 12-lead ECGs}

According to the morphology of different lead of 12-lead ECG PVCs were originated from the LVOT, RVOT (free wall, septal origin, right, left side, proximal, distal origin), basal RV, LV fascicles and aortic cusp (Right and Left coronary cusp). Figure 1 shows a sample of 12-lead ECG of patients with PVC originating from RVOT septum. 

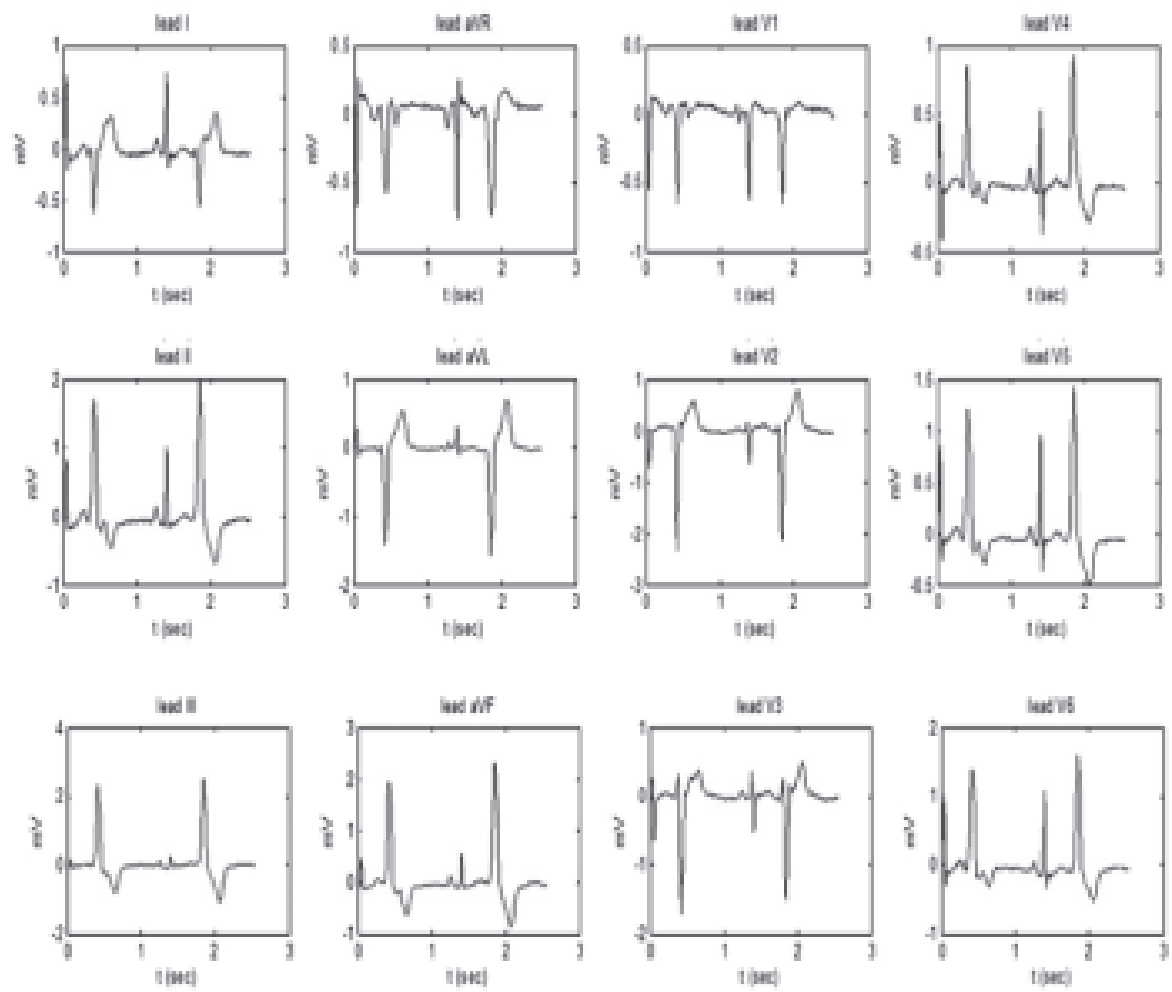

Fig.-1

\section{Results:}

The electrocardiographic characteristics of PVC-QRS morphology of 50 ECGs are shown in Table 1. QRSd d" $140 \mathrm{~ms}$ was $60 \%$, >140ms was $24 \%$, >160ms was $16 \%$. QRS notching $<40 \mathrm{~ms}$ was $42 \%$, $>40 \mathrm{~ms}$ was $16 \%$, smooth contour was $42 \%$. Table II. Showed the morphology of PVCs in lead V1, V2 and lead $1(n=50)$. In lead V1,RBBB morphology was 36\%, LBBB morphology was $64 \%$; in lead V1 \& V2, high r $8 \%$, low r $4 \%$. QRS wave polarity in lead I negative (QS, Qr, or rS wave pattern) 28\%, positive (R-wave pattern) 52\%. Table III. Showed the morphology of PVCs in lead II, III, avF $(n=50)$. Positive 76\%. Of these RR' or Rr' pattern 20\%, R pattern $56 \%$. Negative24\%. Table IV. Showed QRS transition in chest lead $(n=50) .16 \%$ transition occur at $\mathrm{V} 4-\mathrm{V} 5,48 \%$ at $\mathrm{V} 3-\mathrm{V} 4,4 \%$ at $\mathrm{V} 2-\mathrm{V} 3,36 \%$ at $\mathrm{V} 1-\mathrm{V} 2$ level.Table $\mathrm{V}$. showed the pattern of PVCs $(n=50)$. Bigeminy $24 \%$, Trigeminy $6 \%$, Couplet $4 \%$, Salvos $12 \%$, R on T $2 \%$, VT $6 \%$. The sites of origin of all 50 PVCs are shown in Table VI. Of the 32 PVCs originating from the RVOT, 8 were classified as of free-wall origin, 24 of septal, 14 of left, 26 of right, 4 of proximal, and 2 of distal origin. Of the 6 PVCsoriginating from the LVOT, 4 were originated from the LVOT close to the left coronary cusp and 2 were originated from the LVOT close to the right coronary cusp. Of the 12 PVCs originated from LV fascicle, 12 of posterior fascicle origin and none from anterior fascicle origin.

Table-I

Electrocardiographic Characteristics of PVC-QRS morphology $(n=50)$.

\begin{tabular}{lll}
\hline Characteristics & Number & Percentage (\%) \\
\hline
\end{tabular}

QRS duration (ms)

$\begin{array}{ccc}\leq 140 & 30 & 60 \% \\ >140 & 12 & 24 \% \\ >160 & 8 & 16 \%\end{array}$

QRS notching (ms)

$\begin{array}{lcc}<40 & 21 & 42 \% \\ >40 & 8 & 16 \% \\ \text { Smooth contour } & 21 & 42 \%\end{array}$

PVC, premature ventricular complex; ECG, electrocardiogram; < 40ms =narrow notching; $>40 \mathrm{~ms}=$ broad notching. 
Table-II

Morphology of PVCs in lead V1 and lead $1(n=50)$.

\begin{tabular}{lcc}
\hline Morphology in V1 \& V2 & Number & Percent (\%) \\
\hline RBBB & 18 & 36 \\
LBBB & 32 & 64 \\
High $r$ & 4 & 8 \\
Low $r$ & 2 & 4 \\
Morphology in 1 & & \\
Negative & 14 & 28 \\
Positive & 26 & 52 \\
\hline
\end{tabular}

LBBB, Left bundle branch; RBBB, Right bundle branch; High $r$ means initial r-wave amplitude $0.2 \mathrm{mV}$ in both lead; Low r means r-wave amplitude, $0.2 \mathrm{mV}$ in 1 or both leads.

Table-III

Morphology of PVCs in lead II,III,avF ( $n=50)$.

\begin{tabular}{lcc}
\hline Morphology in II, III, avF & Number & Percent (\%) \\
\hline Positive & 38 & 76 \\
RR' or Rr' pattern & 10 & 20 \\
R pattern & 28 & 56 \\
Negative & 12 & 24 \\
\hline
\end{tabular}

Table-IV

QRS transition in chest lead $(n=50)$.

\begin{tabular}{lcc}
\hline QRS transition & Number & Percent $(\%)$ \\
\hline V4-V5 & 8 & 16 \\
V3 -V4 & 24 & 48 \\
V2 -V3 & 2 & 4 \\
V1 -V2 & 18 & 36 \\
\hline
\end{tabular}

Table-V

Pattern of PVCs ( $n=50)$.

\begin{tabular}{lcc}
\hline Pattern & Number & Percent (\%) \\
\hline Bigeminy & 12 & 24 \\
Trigeminy & 3 & 6 \\
Couplet & 2 & 4 \\
Salvos & 6 & 12 \\
R on T & 1 & 2 \\
VT3 & 6 & \\
\hline
\end{tabular}

Table-VI

Number of PVC origins. $(n=50)$

\begin{tabular}{lcc}
\hline Site of origin & Number & Percent (\%) \\
\hline RV outflow tract & 32 & 64.0 \\
Free wall side & 8 & 16.0 \\
Septum side & 24 & 48.0 \\
Left side & 14 & 28.0 \\
Right side & 26 & 52.0 \\
Proximal side below PV & 4 & 8.0 \\
Distal side below PV & 2 & 4.0 \\
LV outflow tract & 6 & 12.0 \\
Left coronary cusp & 4 & 8.0 \\
Right coronary cusp & 2 & 4.0 \\
Fascicular PVC & 12 & 24.0 \\
Posterior fascicle & 12 & 24.0 \\
Anterior fascicle & 0 & 0.0 \\
\hline
\end{tabular}

$\mathrm{RV}$, right ventricle; LV, left ventricle; $\mathrm{PV}$, pulmonary valve.

\section{Discussion:}

This study shows a method to identify PVCs and determine their duration, pattern, and morphology of PVCs in different lead of 12-lead ECG. Through which foci of PVCs can also be determined.

In our study, total 50 ECGs were evaluated. Among them QRSd d" 140 ms was $60 \%$, >140ms was $24 \%$, >160ms was $16 \%$. QRS notching $<40$ ms was $42 \%$, >40ms was $16 \%$, smooth contour was $42 \%$. Broad notches or shelves coupled with QRS duration $>160$ msec is a useful discriminator between the presence and absence of heart disease, as suggestedby other studies [4, 5].These PVCs might serve as a reliable marker for a particular structural and functional state of a nonspecifically diseased myocardium: dilated and globally hypokinetic. On the other hand, PVCs (with smooth contour or narrow notches as well as QRS duration $<160 \mathrm{msec}$ is more likely to identify patients with normal-size hearts with normal or near-normal left ventricular function despite the presence of underlying cardiac disease ${ }^{13}$.

We also evaluated morphology of PVCs in different leads of 12-lead ECG, like lead I, II, III, avF, V1 and QRS transition in precordial leads. Through which location of PVCs origins can be determined.

In our study, 32 PVCs (64\%) were originated from RVOT, which is consistent with previous study ${ }^{9}$. RVOT PVC is associated with a characteristic ECG morphology of LBBB in lead V1 with inferior axis ${ }^{11}$. The QRS duration and the QRS wave morphology in leads II and III is informative. If the QRS duration is $>140 \mathrm{~ms}$, the origin is likely to be on the free-wall side. If it is d"140 ms, the origin is likely to be on the septal side (diagnostic accuracy: $80 \%$ ). If the RR' or Rr' wave pattern is observed in leads II and III, the origin is on the free-wall side. If the R-wave pattern was seen in leads II and III, the origin was likely to be on the septal side (diagnostic accuracy: 86\%). QRS transition at V4-V5, origin 
is free-wall RVOT and transition at V3-V4, origin is septal RVOT. The QRS wave polarity of lead I is another useful index. If lead I showed negative polarity (QS, Qr, or rS wave pattern), the origin is likely to be on the left side. If lead I showed positive polarity (R-wave pattern), the origin is likely to be on the right side (diagnostic accuracy: $83 \%$ ).If the initial r-wave amplitude in leads $\mathrm{V} 1$ and $\mathrm{V} 2$ is high (e"0.2 $\mathrm{mV}$ in both leads), the origin is likely to be on the proximal side. If the initial r-wave amplitude in V1 and V2 is low ( $<0.2 \mathrm{mV}$ in one or both leads), the origin is likely to be on the distal side (diagnostic accuracy: 66\%) ${ }^{12}$.

LVOT PVC is suggested by RBBB morphology in lead V1 with inferior axis or LBBB morphology with inferior axis with small R-waves in V1 and early precordial transition (R/S = 1 by V2 or V3) [11]. In our study, 6 PVCs (12\%) were originated from LVOT, which is also consistent with previous study [9]. Among them 4 were originated from the LVOT close to the left coronary cusp and 2 were originated from the LVOT close to the right coronary cusp. Aortic sinus cusp origin is sometimes difficult to differentiate from RVOT PVC because both are so close to each other. VT/PVC originated from the left coronary cusp produces multiphasic QRS morphology with an M or $\mathrm{W}$ configuration in lead $\mathrm{V} 1$ with a precordial transition no later than V2. A left bundle pattern with a wide small $R$ wave in lead V2 and a precordial transition usually at V3 is revealed in PVCs with a right coronary cusp origin ${ }^{6}$. The RBBB QRS configuration with a left superior axis, suggesting an exit site from the posterior fascicle and RBBB/right inferior axis, suggesting an exit site from anterior fascicle. In our study, we found of the 12 PVCs originated from LV fascicle, 12 from posterior fascicle origin and none from anterior fascicle origin.

We also found several pattern of PVCs like bigeminy, trigeminy, couplet, salvos, R on T, VT in our study.

The clinical value of studying morphology of PVCs in 12lead ECG is a cost effective and noninvasive means of risk stratifying patients early during the initial evaluation period when used in conjunction with the history and physical examination. It can also serve to prompt additional caution when contemplating the use of drugs that significantly impair ventricular function, including certain antiarrhythmic agents. Studying morphology of PVCs in different lead of 12-lead ECG also helps determining their foci and can be important for ablation procedure and may help to improve ablation outcome.

\section{References:}

1. Chiu CC, Lin TH, Liau BY: Using correlation coefficient in ECG waveform for arrhythmia detection. Biomed EngApp, Bas C 2005, 17: 14752. $10.4015 / \mathrm{S} 1016237205000238$ CrossRef

2. Chikh MA, Ammar M, Marouf R: A neuro -fuzzy identification of ECG beats. J Med Syst 2010. Doi: $10.1007 / \mathrm{s} 10916-010-9554-4$
3. Galen S. Wagner, David G. Strauss. Marriott's Practical Electrocardiography. In: Galen S. Wagner. Premature beats. $12^{\text {th }}$ edition: $2014: 15 ; 324-34$.

4. Soloff LA: Ventricular premature beats diagnostic of myocardial disease. Am J Med Sci 1961;242:315-319

5. Scherf D, Schott A: Extrasystoles and Allied Arrhythmias. NewYork, Grune \& Stratton, Inc, 1953, p 30

6. Lin D, Ilkhanoff L, Gerstenfeld E, Dixit S, Beldner S, Bala R, Garcia F, Callans D, Marchlinski FE: Twelvelead electrocardiographic characteristics of the aortic cusp region guided by intracardiac echocardiography and electroanatomic mapping. Heart Rhythm Society 2008, 5: 663-9. 10.1016/j. hrthm.2008.02.009CrossRef

7. Betensky BP, Park RE, Marchlinski FE, et al.: A new electrocardiographic criterion for distinguishing left from right ventricular outflow tract tachycardia origin FREE. J Am Coll Cardiol 2011, 57(22):2255-62. doi:10.1016/j.jacc.2011.01.035 10.1016/j.jacc. 2011.01.035CrossRef

8. Nathani P, Shetty S, Lokhandwala Y: Ventricular tachycardia in structurally normal hearts: recognition and management. Supplement of $\mathrm{J}$ Assoc Physicians India 2007, 55(suppl):33-8.

9. Lerman BB, Stein KM, Markowitz SM: Mechanism of idiopathic left ventricular tachycardia. J Cardiovasc Electrophysiol 1997, 8(5):571-83. 10.1111/j.15408167.1997.tb00826.xCrossRef

10. Nakagawa M, Takahashi N, Nobe S, et al.: Gender differences in various types of idiopathic ventricular tachycardia. J Cardiovasc Electrophysiol 2002, 13: 633-8.10.1046/j.1540-8167.2002.00633. xCross Ref

11. Shin SY, Joo HJ, Kim JH, Jang JK, Park JS, Kim YH, Lee HS, Choi JI, Lim HE, Kim YH: Epicardial conduction properties and electrocardiographic characteristics of premature ventricular complexes or ventricular tachycardias that originate at the aortic cusp. Korean Circ J 2007, 37: 616-22. 10.4070/ kcj.2007.37.12.616CrossRef

12. Kamakura S, Shimizu W, Matsuo K, Taguchi A, Suyama K, Kurita T, Aihara N, Ohe T, Shimomura K: Localization of optimal ablation site of idiopathic ventricular tachycardia from right and left ventricular outflow tract by body surface ECG. ( 1998 Am Heart Assoc 1998, 98: 1525-33. Inc.Circulation1998

13. Kriegh P. Moulton, MD, Tim Medcalf, MD, and Ralph Lazzara, MD: Premature Ventricular Complex Morphology. A Marker for Left Ventricular Structure and Function.Circulation 1990;81:1245-51 